Iowa State University

Digital Repository @ Iowa State University

NCRPIS Publications and Papers

North Central Regional Plant Introduction Station

$2-27-2014$

Reassessment of Practical Subspecies Identifications of the USDA Daucus carota L. Germplasm Collection: Morphological Data

David M. Spooner

United States Department of Agriculture

Mark P. Widrlechner

Iowa State University, isumw@iastate.edu

Kathleen R. Reitsma

Iowa State University, kreitsma@iastate.edu

Debra E. Palmquist

United States Department of Agriculture

Slim Rouz

Followsthis and additional,works at: http://lib.dr.iastate.edu/ncrpis_pubs

Part of the Agricultural Science Commons, Agriculture Commons, Horticulture Commons, and the next pant Breeding and Genetics Commons

The complete bibliographic information for this item can be found at http:/ /ib.dr.iastate.edu/ ncrpis_pubs/41. For information on how to cite this item, please visit http://lib.dr.iastate.edu/ howtocite.html.

This Article is brought to you for free and open access by the North Central Regional Plant Introduction Station at Digital Repository @ Iowa State University. It has been accepted for inclusion in NCRPIS Publications and Papers by an authorized administrator of Digital Repository @ Iowa State University. For more information, please contact digirep@iastate.edu. 


\section{Authors}

David M. Spooner, Mark P. Widrlechner, Kathleen R. Reitsma, Debra E. Palmquist, Slim Rouz, Zeineb Ghrabi-Gammar, Mohamed Neffati, Béchir Bouzbida, Philipp W. Simon, and Mohammed El Koudrim 


\title{
Reassessment of Practical Subspecies Identifications of the USDA Daucus carota L. Germplasm Collection: Morphological Data
}

\author{
David M. Spooner, Mark P. Widrlechner, Kathleen R. Reitsma, Debra E. Palmquist, \\ Slim Rouz, Zeineb Ghrabi-Gammar, Mohamed Neffati, Béchir Bouzbida, \\ Hassan Ouabbou, Mohammed El Koudrim, and Philipp W. Simon
}

\begin{abstract}
The genus Daucus includes about 20 recognized species. The most widespread and economically important species, Daucus carota L., occurs on almost every continent. The cultivated carrot, subsp. sativus (Hoffm.) Schübl. and G. Martens, has been selected from wild populations that are extremely diverse, especially in the western Mediterranean. The predominant outcrossing and the lack of sexual isolating mechanisms among recognized infraspecific taxa complicate the taxonomy and identification of the wild populations, resulting in widely different interpretations of the number of infraspecific taxa. We measured 36 morphological characters from multiple individuals within each of 155 accessions of $D$. carota and from the morphologically similar species $D$. capillifolius (both species $2 n=18$ ) alongside other species for comparison ( $D$. aureus Desf., $2 n=22$; $D$. broteri Ten., $2 n=20$; $D$. involucratus Sm., $2 n=20$; and $D$. littoralis $\mathrm{Sm}$., $2 n=20$ ) in an experimental field plot. Within $D$. carota, multivariate analyses were able to identify only two subspecies, but even these showed great overlap of individual characters. Because of the ease of crossability of wild $D$. carota to the domestic landraces and cultivars and because of the taxonomic challenges, the purpose of our study is to explore morphological support for subspecies within $D$. carota, including the phenetically similar $D$. capillifolius, which is part of the same clade as $D$. carota, with the long-term goal of resolving taxonomic disagreements and developing a practical system to classify variation within this economically important species.
\end{abstract}

D.M. Spooner and P.W. Simon, USDA-ARS, Dep. of Horticulture, Univ. of Wisconsin, 1575 Linden Dr., Madison, WI 53706-1590; M.P. Widrlechner, Iowa State Univ., Dep. of Horticulture and Dep. of Ecology, Evolution, and Organismal Biology, 251 Bessey Hall, Ames, IA 50011; K.R. Reitsma, Iowa State Univ., North Central Regional Plant Introduction Station, Ames, IA 50011; D.E. Palmquist, USDA-ARS, Midwest Area Office, 1815 N. University St., Peoria, IL 61604; S. Rouz, Institut Supérieur Agronomique de Mogran, Tunisia; Z. Ghrabi-Gammar, Institut National Agronomique de Tunisie, Tunisia; M. Neffati and B. Bouzbida, Institut des Régions Arides, 4119 Medenine, Tunisia; and H. Ouabbou and M. El Koudrim, Institut National de la Recherche Agronomique, CRRA de Settat, B.P. 589, Settat 26000, Morocco. Received 8 Apr. 2013. ^Corresponding author (david.spooner@ars.usda.gov).

Abbreviations: GRIN, Germplasm Resources Information Network; NCRPIS, North Central Regional Plant Introduction Station.

$\mathrm{T}$ HE Umbelliferae comprise some 300-455 genera and 30003750 species (Constance, 1971; Pimenov and Leonov, 1993). The family is cosmopolitan but most diverse in the Northern Hemisphere, particularly in the Mediterranean region. The cultivated carrot (Daucus carota L. subsp. sativus) is by far its economically most important member, but the family also contains many other vegetables, flavorings, and herbs, including angelica, anise, caraway, celeriac, celery, chervil, coriander (cilantro), cumin, dill, fennel, lovage, parsley, and parsnip (Rubatzky et al., 1999).

While it is easy to place species within the Umbelliferae, existing generic boundaries within this large family are unnatural. Recent molecular investigations based on DNA sequences from nuclear ribosomal internal transcribed spacers, plastid rpoC1 intron and rpl16 intron sequences, plastid mat $K$ coding sequences,

Published in Crop Sci. 54:706-718 (2014).

doi: 10.2135/cropsci2013.04.0231

(C) Crop Science Society of America | 5585 Guilford Rd., Madison, WI 53711 USA

All rights reserved. No part of this periodical may be reproduced or transmitted in any form or by any means, electronic or mechanical, including photocopying, recording, or any information storage and retrieval system, without permission in writing from the publisher. Permission for printing and for reprinting the material contained herein has been obtained by the publisher. 
plastid DNA restriction-site data, and DNA sequences from nuclear orthologs (Plunkett et al., 1996; Downie et al., 2000; Lee and Downie, 2000; Spalik and Downie, 2007; Spooner et al., 2013) do not support many genera within the Umbelliferae as monophyletic. This is clearly the case with Daucus, as molecular data from these papers above place species from the genera Agrocharis, Athamanta, Cryptotaenia, Margotia, Melanoselinum, Monizia, Pachyctenium, Pseudorlaya, and Tornabenea within a monophyletic Daucus clade.

The latest taxonomic monograph of Daucus was by Sáenz Laín (1981), who recognized 20 species. The haploid chromosome number for Daucus ranges from $n=9$ to $n=11$. Most species are diploids with $2 n=20$ or $2 n=22$, but two tetraploid species have been reported (Grzebelus et al., 2011). There are only four $2 n=18$ chromosome species of Daucus: the widespread D. carota (all subspecies) and three North African species-D. capillifolius Gilli, D. syrticus Murb., and D. sahariensis Murb. (Grzebelus et al., 2011). DNA sequencing data of multiple nuclear orthologs (Arbizu et al., 2013; Spooner et al., 2013) place all subspecies of D. carota and D. capillifolius as a monophyletic clade, with $D$. sahariensis and $D$. syrticus as sisters to this clade. Daucus carota and D. capillifolius, but excluding $D$. sahariensis and $D$. syrticus (here referred to as the $D$. carota clade, or the $2 n=18$ accessions), are clearly interrelated based on the molecular studies mentioned above, shared karyotypes (Iovene et al., 2008), and crossability data (McCollum, 1975, 1977). Daucus carota is strongly outcrossing, and its populations are genetically heterogeneous (Simon, 1984). All known crosses among the subspecies of $D$. carota and D. capillifolius are interfertile, as evidenced by the results of manual crosses (Krick1, 1961; McCollum, 1975, 1977; Umiel et al., 1975; Ellis et al., 1993; Steinborn et al., 1995; Nothnagel et al., 2000). In addition, morphological intermediates among sympatric subspecies of $D$. carota are common and have been ascribed to natural intersubspecific hybridization (Nehou, 1961; Heywood, 1968; Wijnheijmer et al., 1989; Magnussen and Hauser, 2007).

This ease of crossing and great morphological variation within $D$. carota have resulted in confusing patterns of natural variation and widely different classifications. Within D. carota, two groups are phenotypically coherent: (i) plants with a relatively short stature, thick, broad leaf segments, and usually flat or convex fruiting umbels, distributed in the coastal regions of the central and western Mediterranean and Atlantic coasts of northern Africa, Portugal, Spain, France, and the UK; and (ii) taller plants with thinner narrower leaf segments and fruiting umbels that are frequently curved upward and that close into a characteristic "bird's nest" form, occurring in coastal regions as above but also in inland regions and over a greater distributional range that includes Asia, Australia, and the Americas. Onno (1937) classified populations of the first group as D. gingidium L., containing eight subspecies, and the latter as D. carota, including four subspecies. Small (1978) and
Reduron (2007) recognized two "species aggregates," or "subgroups,"within the single species D. carota corresponding to the above two groups. Reduron (2007) recognized five species within subgroup carota and four subspecies within subgroup gummifer, our first group above. Heywood (1968), Sáenz Laín (1981), and Pujadas Salvà (2003) recognized only a single species, but without the division into subgroups. They divided D. carota into subspecies but differed in the number of their recognized subspecies.

Daucus identifications made at the USDA-ARS North Central Regional Plant Introduction Station (NCRPIS) in Ames, Iowa, have typically been based on the sole comprehensive taxonomic treatment by Sáenz Laín (1981) supplemented by floristic treatments, such as those from Algeria (Quezel and Santa, 1963), Europe (Heywood, 1968), the Iberian Peninsula and Balearic Islands (Pujadas Salvà, 2003), Libya (Jafri and El-Gadi, 1985), Morocco (Jury, 2002), Tunisia (Le Floc'h et al., 2010), Palestine (Zohary, 1972), Syria (Mouterde, 1986), and Turkey and the East Aegean Islands (Cullen, 1972). However, identifications in these taxonomic treatments frequently use different characters and character states in their taxonomic keys and descriptions; have incomplete synonymies, which preclude comparison of their taxonomic concepts; often have little information about geographic ranges; and lack distribution maps. In addition, there has been no single compilation of type specimens, and many of the types lack the full range of plant parts necessary for unambiguous identification. In summary, there has been no accepted standard to quantify and describe the huge range of variation in D. carota, and identification of the accessions conserved by the NCRPIS is often problematic.

The NCRPIS conserves 1381 accessions of Daucus. Of these, 566 are classified as landraces, cultivars, cultivated populations, or breeding lines. Improvement status for the remaining accessions include 570 wild, 17 uncertain, and 227 accessions have no status designated (though many of these most likely are cultivated). Taxonomically, there are 917 accessions identified as D. carota, with 247 of these identified as $D$. carota with a variety or subspecies designation (1164 D. carota total), leaving 217 accessions identified as other Daucus species.

Because of the ease of crossability of wild D. carota to the domestic landraces and cultivars, and because of the taxonomic challenges noted earlier, the purpose of our study was to explore morphological support for various subspecies within $D$. carota, with the long-term goal of developing a practical system to classify variation within this economically important species. To this end, we also included D. capillifolius because it shares the same chromosome number $(2 n=18)$ and crossability pattern as $D$. carota and because it is part of the $D$. carota clade (Arbizu et al., 2013; Spooner et al., 2013). We also included four morphologically distinct species of Daucus with different base chromosome numbers as comparator species. 


\section{MATERIALS AND METHODS Accessions Examined}

We examined a total of 155 accessions of $D$. carota, all of wild origin except for one accession of $D$. carota subsp. sativus var. atrorubens Alef., including those with no subspecies designation and those previously identified as subsp. carota, subsp. commutatus (Paol.) Thell., subsp. drepanensis (Arcang.) Heywood, subsp. fontanesii Thell., subsp. gummifer (Syme) Hook.f., subsp. hispanicus (Gouan) Thell., subsp. major (Vis.) Arcang., subsp. maritimus (Lam.) Batt., subsp. maximus (Desf.) Ball, D. capillifolius, and putative interspecific hybrids between $D$. capillifolius and D. carota subsp. carota (all above presumed to be $2 n=18$, based on identifications of these accessions). For comparison, we also examined accessions morphologically distinctive from $D$. carota and $D$. capillifolius and outside of the $D$. carota clade, including D. aureus Desf. $(2 n=22)$, D. broteri Ten. $(2 n=20)$, D. guttatus Sibth. and Sm. $(2 n=20)$, D. involucratus Sm. $(2 n=20)$, and D. littoralis $\mathrm{Sm}$. $(2 n=20)$. We measured different accessions in 2010 and 2011; 15 replicates were measured in both years (Table 1), raising the total from 155 to 170 examinations.

\section{Daucus Observation Plots}

Different accessions were measured in 2010 and 2011 except that 15 accessions were measured in both years to ensure that character development was consistent in different years (Table 1). For the 2010 observations, one 6-m row of each accession was direct seeded in field plots using a V-belt push planter with 3-m alleys between rows. Accessions were thinned to 20 plants per row, and traits (descriptors) were measured on at least three plants per accession. Plant and umbel descriptor data were collected during the growing season. Field plots were maintained with small plot tillers and hand weeding.

To better ensure sufficient plant populations in the 2011 observation plot, biennial and mixed life-cycle accessions were planted in the greenhouse in early November 2010. Seedlings were thinned to one per pot, and plants were fertilized weekly with a commercial liquid fertilizer (NPK 20-10-20). Roots were vernalized in the dark $\left(4-5^{\circ} \mathrm{C}, 50-70 \%\right.$ relative humidity) for approximately $60 \mathrm{~d}$ beginning in February 2011. A fungicide spray (Rubigan, DuPont, Wilmington, DE) was applied at the beginning of vernalization and reapplied as necessary to prevent Botrytis blight. Roots were moved outside to a protected area in mid-April to allow them to develop new foliage. Twenty plants per accession were transplanted into 6-m rows, one row per accession in each of two field plots in early May. Annual accessions were direct seeded into two field plots as described for the 2010 observation plot. Field plots were maintained with small plot tillers and hand weeding.

\section{Characters Measured}

Thirty-six characters were measured from at least three individuals per accession (Table 2). These characters were chosen to represent all those used in prior keys and morphological analyses (Small, 1978) to distinguish subspecies within D. carota and between $D$. carota and morphologically similar species. Size characters were measured in the field with a ruler or calipers, and floral and fruit characters were measured in the laboratory with the aid of a dissecting microscope. For both year's observations, electronic images of leaves were generated on a flatbed scanner; images of various plant parts were made from plants in the field with a digital camera; and all images are available on the Germplasm Resources Information Network (GRIN; www.ars-grin.gov/). Herbarium vouchers and alcohol-preserved inflorescences in flower and fruit of all of the accessions are deposited at the herbarium of the Potato Introduction Station, Sturgeon Bay, WI.

\section{Analytical Methods}

Thirty-two of the 36 characters were scored and analyzed as continuous variables; the remaining four were treated as nominal variables (Table 2). Means were taken of the former and modes for the latter. All analyses were conducted in JMP 9.0.3 software (SAS Institute, 2010). We ran two types of analyses to explore the best ways to distinguish the groups. We first performed hierarchical cluster analyses, all with standardized data, exploring five distance methods: average, centroid, ward, single, and complete. Second, we performed stepwise discriminant analyses (linear, common covariance) using all 32 continuous variables to obtain a model whose variables were significant in correctly identifying accession composition, with characters removed one at a time until the model F-test $p$ value was $\leq 0.05$. This process was conducted in three iterations until a combination of reidentifications resulted in all taxa being confidently identified by this method, but with reidentifications verified only after examination of herbarium vouchers and photographs of the accessions. Once the taxa were reidentified (Table 1), we conducted stepwise discriminant analysis of all taxa (Fig. 1), and subsequently with all $2 n=18$ taxa only (Fig. 2). Histograms were then constructed to show character-state distributions of the 10 characters exhibiting the highest F-values (all with $p \leq 0.05$ ) within each of the above two methods (Fig. 3 and 4).

\section{Field Observations in Morocco, Tunisia, and the United States}

In addition to our common-garden studies, field trips to collect Daucus germplasm from wild and weedy populations were made by coauthors Simon and Spooner to Tunisia in 2009, the western United States in 2010 and 2011, and Morocco in 2012 and 2013. Daucus carota was collected extensively on all trips, and numerous collections of D. capillifolius made in Tunisia. Trips were taken in August or September in 2009-2012, when we could observe late-flowering and mature-fruiting plants, and in June 2013 for flowering plants in Morocco. Daucus carota and $D$. capillifolius generally occur in disturbed areas along roadsides, in cultivated fields, and in peri-urban locales. Plant populations were frequently very large near the Mediterranean Sea (Tunisia and Morocco) and the Pacific Ocean (Washington, Oregon, and California of the United States) and also inland where natural rainfall was relatively plentiful or near irrigated agriculture. Populations reidentified as D. carota subsp. gummifer were all confined to areas within a few kilometers of the Mediterranean Sea or the Pacific Ocean. 
Table 1. The 170 accessions (including one landrace, $D$. carota var. atrorubens) of wild carrot examined in this study, the year examined, the generalized locality, and original and proposed new identifications.

\begin{tabular}{|c|c|c|c|c|}
\hline $\begin{array}{c}\text { Original } \\
\text { identification }\end{array}$ & Accession & Year & Location of collection $^{\dagger}$ & $\begin{array}{l}\text { Proposed new } \\
\text { identifications }^{\ddagger}\end{array}$ \\
\hline Daucus aureus & PI 295854 & 2010 & Israel, Wadi Rubin (HaMerkaz) & \\
\hline D. aureus & PI 319403 & 2010 & Israel, Mediterranean region & \\
\hline D. aureus & PI 478858 & 2010 & France, Dijon & \\
\hline D. broteri & Ames 25721 & 2010 & Syria, Younesiya & \\
\hline D. broteri & Ames 25729 & 2010 & Syria, Qastal & \\
\hline D. broteri & PI 652329 & 2010 & Greece, Peloponnese & \\
\hline D. broteri & PI 652385 & 2010 & Turkey, Antalya & \\
\hline D. capillifolius & Ames 30198 & 2010 & Tunisia, Medenine & \\
\hline D. capillifolius & Ames 30225 & 2010 & Sfax, Tunisia & \\
\hline D. capillifolius & Ames 30233 & 2010 & Tunisia, Mahdia & \\
\hline D. capillifolius & PI 279764 & 2010 & Libya, near Jefren & \\
\hline D. carota & Ames 25612 & 2010 & Greece, Macedonia & D. carota subsp. carota \\
\hline D. carota & PI 242384 & 2011 & USA, Maryland & D. carota subsp. carota \\
\hline D. carota & PI 242385 & 2011 & USA, Maryland & D. carota subsp. carota \\
\hline D. carota & PI 274298 & 2011 & Pakistan, Parachinar & D. carota subsp. carota \\
\hline D. carota & PI 287518 & 2011 & Iran, Khoiy & D. carota subsp. carota \\
\hline D. carota & PI 344446 & 2011 & Iran, Khoiy & D. carota subsp. carota \\
\hline D. carota & PI 344447 & 2011 & Iran, Hamadan & D. carota subsp. gummifer \\
\hline D. carota & PI 652213 & 2011 & Kazakhstan, Chimkent & D. carota subsp. carota \\
\hline D. carota & PI 652214 & 2011 & Portugal, Peso da Regua & D. carota subsp. carota \\
\hline D. carota & PI 652215 & 2011 & USA, Colorado & D. carota subsp. carota \\
\hline D. carota & PI 652219 & 2011 & Hungry, Lake Balaton & D. carota subsp. carota \\
\hline D. carota & PI 652220 & 2011 & Poland, Chelm & D. carota subsp. carota \\
\hline D. carota & PI 652221 & 2011 & Poland, Lublin & D. carota subsp. carota \\
\hline D. carota & PI 652222 & 2011 & Portugal, Vila Real & D. carota subsp. carota \\
\hline D. carota & PI 652223 & 2011 & Poland, Nowy Sacz & D. carota subsp. carota \\
\hline D. carota & PI 652224 & 2011 & Poland, Lomza & D. carota subsp. carota \\
\hline D. carota & PI 652292 & 2011 & Greece, Macedonia & D. carota subsp. carota \\
\hline D. carota & PI 652295 & 2011 & Greece, Epirus & D. carota subsp. carota \\
\hline D. carota & PI 652297 & 2011 & Greece, Epirus & D. carota subsp. carota \\
\hline D. carota & PI 652299 & 2011 & Greece, Ionian Islands & D. carota subsp. carota \\
\hline D. carota & PI 652301 & 2011 & Greece, Ionian Islands & D. carota subsp. carota \\
\hline D. carota & PI 652304 & 2011 & Greece, Peloponnese & D. carota subsp. carota \\
\hline D. carota & PI 652306 & 2011 & Greece, Peloponnese & D. carota subsp. carota \\
\hline D. carota & PI 652309 & 2011 & Greece, Peloponnese & D. carota subsp. carota \\
\hline D. carota & PI 652311 & 2011 & Greece, Central Greece & D. carota subsp. carota \\
\hline D. carota & PI 652313 & 2011 & Greece, Central Greece & D. carota subsp. carota \\
\hline D. carota & PI 652335 & 2011 & Syria, Damascus & D. carota subsp. carota \\
\hline D. carota & PI 652344 & 2011 & Syria, Alratbeh & D. carota subsp. carota \\
\hline D. carota & PI 652346 & 2011 & Syria, Crac des Chevaliers & D. carota subsp. carota \\
\hline D. carota & PI 652347 & 2011 & Syria, Sweida & D. carota subsp. carota \\
\hline D. carota & PI 652348 & 2011 & Turkey, Izmir & D. carota subsp. carota \\
\hline D. carota & PI 652353 & 2011 & Turkey, Izmir & D. carota subsp. carota \\
\hline D. carota & PI 652358 & 2011 & Turkey, Izmir & D. carota subsp. carota \\
\hline D. carota & PI 652361 & 2011 & Turkey, Mugla & D. guttatus \\
\hline D. carota & PI 652364 & 2011 & Turkey, Mugla & D. carota subsp. carota \\
\hline D. carota & PI 652369 & 2011 & Turkey, Mugla & D. carota subsp. carota \\
\hline D. carota & PI 652373 & 2011 & Turkey, Mugla & D. carota subsp. carota \\
\hline D. carota & PI 652375 & 2011 & Turkey, Mugla & D. guttatus \\
\hline D. carota & PI 652378 & 2011 & Turkey, Mugla & D. carota subsp. carota \\
\hline D. carota & PI 652384 & 2011 & Turkey, Antalya & D. carota subsp. carota \\
\hline D. carota & PI 652395 & 2011 & Turkey, Konya & D. guttatus \\
\hline D. carota & PI 652398 & 2011 & Turkey, Isparta & D. carota subsp. carota \\
\hline D. carota & PI 652406 & 2011 & Turkey, Denizli & D. carota subsp. carota \\
\hline
\end{tabular}


Table 1. Continued.

\begin{tabular}{|c|c|c|c|c|}
\hline $\begin{array}{c}\text { Original } \\
\text { identification }\end{array}$ & Accession & Year & Location of collection $^{\dagger}$ & $\begin{array}{l}\text { Proposed new } \\
\text { identifications }{ }^{\ddagger}\end{array}$ \\
\hline D. carota & PI 652407 & 2011 & Turkey, Denizli & D. carota subsp. carota \\
\hline D. carota & PI 652408 & 2011 & Turkey, Denizli & D. guttatus \\
\hline D. carota subsp. carota & Ames 25740 & 2010 & Syria, As Samra & \\
\hline D. carota subsp. carota & Ames 26371 & 2011 & Portugal, Braga & \\
\hline D. carota subsp. carota & Ames 26372 & 2011 & Portugal, Braga & \\
\hline D. carota subsp. carota & Ames 26376 & 2010 & Portugal, Castelo Branco & \\
\hline D. carota subsp. carota & Ames 27397 & 2010 & Uzbekistan, between Yalangoch and Sobir Raximova & \\
\hline D. carota subsp. carota & Ames 27401 & 2010 & Uzbekistan, Tashkent to Bolta & \\
\hline D. carota subsp. carota & Ames 27403 & 2010 & Uzbekistan, Angren to Tashkent & \\
\hline D. carota subsp. carota & Ames 27410 & 2010 & Uzbekistan, Between Kitab and Samarkand & \\
\hline D. carota subsp. carota & Ames 27416 & 2010 & Uzbekistan, between Angren and Nurobad & \\
\hline D. carota subsp. sativus & Ames 30234 & 2010 & Tunisia landrace & \\
\hline D. carota subsp. carota & Ames 30242 & 2010 & Tunisia, Ben Arous & \\
\hline D. carota subsp. carota & Ames 30244 & 2010 & Tunisia, Zaghouan & \\
\hline D. carota subsp. carota & Ames 30245 & 2010 & Tunisia, Zaghouan & \\
\hline D. carota subsp. carota & Ames 30248 & 2010 & Tunisia, Zaghouan & $\begin{array}{l}\text { Daucus hybrid } \\
\text { (D. carota, D. capillifolius) }\end{array}$ \\
\hline D. carota subsp. carota & Ames 30249 & 2010 & Tunisia, Nabeul & \\
\hline D. carota subsp. carota & Ames 30256 & 2010 & Tunisia, L’Ariana & \\
\hline D. carota subsp. carota & Ames 30258 & 2010 & Tunisia, Bizerte & \\
\hline D. carota subsp. carota & Ames 30262 & 2010 & Tunisia, Beja & \\
\hline D. carota subsp. carota & Ames 30265 & 2010 & Tunisia, Jendouba & \\
\hline D. carota subsp. carota & Ames 30272 & 2010 & Tunisia, Jendouba & \\
\hline D. carota subsp. carota & PI 274297 & 2010 & Pakistan, Northern Areas & \\
\hline D. carota subsp. carota & PI 279788 & 2010 & Austria, Vienna & \\
\hline D. carota subsp. carota & PI 295861 & 2010 & Spain, El Viso & \\
\hline D. carota subsp. carota & PI 390887 & 2010 & Israel, central Israel & \\
\hline D. carota subsp. carota & PI 421301 & 2010 & USA, Kansas & \\
\hline D. carota subsp. carota & PI 430525 & 2010 & Afghanistan, Zardek & \\
\hline D. carota subsp. carota & PI 478369 & 2010 & China, Xinjiang & \\
\hline D. carota subsp. carota & PI 478859 & 2010 & Italy, Rimini & \\
\hline D. carota subsp. carota & PI 478860 & 2010 & France, Seine et Oise & \\
\hline D. carota subsp. carota & PI 478861 & 2010 & France, Seine et Oise & \\
\hline D. carota subsp. carota & PI 478863 & 2010 & Collection site unknown & \\
\hline D. carota subsp. carota & PI 478869 & 2010 & Germany, Juterbog & \\
\hline D. carota subsp. carota & PI 478873 & 2010 & Italy, Sardinia & \\
\hline D. carota subsp. carota & PI 478875 & 2010 & Italy, Molise & \\
\hline D. carota subsp. carota & PI 478876 & 2010 & Italy, Latium & \\
\hline D. carota subsp. carota & PI 478878 & 2010 & Switzerland, Geneva & \\
\hline D. carota subsp. carota & PI 478881 & 2010 & USA, Oregon & \\
\hline D. carota subsp. carota & PI 478884 & 2010 & The Netherlands, South Holland & \\
\hline D. carota subsp. carota & PI 652191 & 2010 & Poland, Okolice & \\
\hline D. carota subsp. carota & PI 652218 & 2010 & Hungary, Békés & \\
\hline D. carota subsp. carota & PI 652236 & 2010 & Bulgaria, Lovech & \\
\hline D. carota subsp. carota & PI 652296 & 2010 & Greece, Epirus & \\
\hline D. carota subsp. carota & PI 652303 & 2010 & Greece, Central Greece & \\
\hline D. carota subsp. carota & PI 652320 & 2010 & Greece, Macedonia & \\
\hline D. carota subsp. carota & PI 652341 & 2010 & Syria, Ash Sheik Hasan & \\
\hline D. carota subsp. carota & PI 652351 & 2010 & Turkey, Izmir & \\
\hline D. carota subsp. carota & PI 652379 & 2010 & Turkey, Mulga & \\
\hline D. carota subsp. carota & PI 652393 & 2010 & Turkey, Konya & \\
\hline D. carota subsp. carota & PI 652409 & 2010 & Turkey, Aydin & \\
\hline carota subsp. commutatus & Ames 7674 & 2011 & Italy, Tuscany & D. carota subsp. gummifer \\
\hline carota subsp. commutatus & PI 478883 & 2010 & France, Finistere & D. carota subsp. gummifer \\
\hline carota subsp. commutatus & PI 652291 & 2010 & Portugal, Faro & D. carota subsp. gummifer \\
\hline
\end{tabular}




\begin{tabular}{|c|c|c|c|c|}
\hline $\begin{array}{c}\text { Original } \\
\text { identification }\end{array}$ & Accession & Year & Location of collection $^{\dagger}$ & $\begin{array}{l}\text { Proposed new } \\
\text { identifications }^{\ddagger}\end{array}$ \\
\hline D. carota subsp. drepanensis & PI 279794 & 2010 & Spain, Madrid & D. carota subsp. gummifer \\
\hline D. carota subsp. fontanesii & PI 652387 & 2010 & Turkey, Antalya & D. guttatus \\
\hline D. carota subsp. gummifer & Ames 26382 & 2010 and 2011 & Portugal, Faro & \\
\hline D. carota subsp. gummifer & Ames 26383 & 2010 and 2011 & Portugal, Faro & \\
\hline D. carota subsp. gummifer & Ames 26384 & 2010 and 2011 & Portugal, Beja & \\
\hline D. carota subsp. gummifer & PI 652411 & 2010 & France, Finistere & \\
\hline D. carota subsp. hispanicus & PI 652139 & 2010 & Italy, Apulia & D. carota subsp. gummifer \\
\hline D. carota subsp. hispanicus & PI 652150 & 2010 & Collection site unknown & D. carota subsp. gummifer \\
\hline D. carota subsp. major & Ames 24682 & 2010 and 2011 & Portugal, Coimbra & D. carota subsp. carota \\
\hline D. carota subsp. major & Ames 25017 & 2010 and 2011 & Germany, Saxony-Anhalt & D. carota subsp. carota \\
\hline D. carota subsp. major & Ames 25898 & 2011 & Turkey, Konya & D. carota subsp. carota \\
\hline D. carota subsp. major & PI 652226 & 2011 & Greece, $10 \mathrm{~km} N$ of Kassandra & D. carota subsp. carota \\
\hline D. carota subsp. major & PI 652229 & 2011 & Tunisia & D. carota subsp. carota \\
\hline D. carota subsp. maritimus & Ames 26386 & 2011 & Portugal, Braganca & D. carota subsp. carota \\
\hline D. carota subsp. maritimus & Ames 26387 & 2011 & Portugal, Braganca & D. carota subsp. carota \\
\hline D. carota subsp. maritimus & Ames 26388 & 2011 & Portugal, Braganca & D. carota subsp. carota \\
\hline D. carota subsp. maritimus & Ames 26389 & 2011 & Portugal, Guarda & D. carota subsp. carota \\
\hline D. carota subsp. maritimus & Ames 26393 & 2011 & Portugal, Branco & D. carota subsp. carota \\
\hline D. carota subsp. maritimus & Ames 26398 & 2011 & Portugal, Faro & D. carota subsp. carota \\
\hline D. carota subsp. maritimus & Ames 26399 & 2011 & Portugal, Faro & D. carota subsp. carota \\
\hline D. carota subsp. maritimus & Ames 26400 & 2011 & Portugal, Beja & D. carota subsp. carota \\
\hline D. carota subsp. maritimus & PI 502244 & 2010 and 2011 & Portugal, Coimbra & D. carota subsp. carota \\
\hline D. carota subsp. maritimus & PI 652225 & 2010 and 2011 & Collection site unknown & D. carota subsp. carota \\
\hline D. carota subsp. maximus & Ames 26401 & 2011 & Portugal, Portalegre & D. carota subsp. carota \\
\hline D. carota subsp. maximus & Ames 26403 & 2011 & Portugal, Evora & D. carota subsp. carota \\
\hline D. carota subsp. maximus & Ames 26404 & 2011 & Portugal, Evora & D. carota subsp. carota \\
\hline D. carota subsp. maximus & Ames 26405 & 2011 & Portugal, Beja & D. carota subsp. carota \\
\hline D. carota subsp. maximus & Ames 26406 & 2011 & Portugal, Beja & D. carota subsp. carota \\
\hline D. carota subsp. maximus & Ames 26407 & 2011 & Portugal, Faro & D. carota subsp. carota \\
\hline D. carota subsp. maximus & Ames 26409 & 2011 & Portugal, Faro & D. carota subsp. carota \\
\hline D. carota subsp. maximus & PI 478866 & 2010 and 2011 & Collection site unknown & D. carota subsp. carota \\
\hline D. carota subsp. maximus & PI 478872 & 2010 and 2011 & Germany, Wolferode & D. carota subsp. carota \\
\hline D. carota subsp. maximus & Pl 478874 & 2010 and 2011 & Italy, Sicily & D. carota subsp. carota \\
\hline D. carota subsp. maximus & PI 652227 & 2011 & Croatia, between Hvar and Milna & D. carota subsp. carota \\
\hline D. carota subsp. maximus & PI 652228 & 2011 & Italy, Calabria & D. carota subsp. carota \\
\hline D. carota subsp. maximus & PI 652230 & 2010 and 2011 & Albania, Lushnje & D. carota subsp. carota \\
\hline D. carota var. atrorubens & PI 279777 & 2010 and 2011 & Egypt, Giza & \\
\hline D. guttatus & Ames 25608 & 2010 and 2011 & Greece, Central Greece & \\
\hline D. guttatus & Ames 25724 & 2010 and 2011 & Syria, Younesiya & \\
\hline D. guttatus & Ames 25807 & 2011 & Turkey, Izmir & \\
\hline D. guttatus & PI 279763 & 2011 & Israel, Jerusalem & \\
\hline D. guttatus & PI 652343 & 2011 & Syria, Halwah & \\
\hline $\begin{array}{l}\text { Daucus hybrid } \\
\text { (D. carota, D. capillifolius) }\end{array}$ & Ames 30211 & 2010 & Tunisia, Gabes & D. capillifolius \\
\hline $\begin{array}{l}\text { Daucus hybrid } \\
\text { (D. carota, D. capillifolius) }\end{array}$ & Ames 30215 & 2010 & Tunisia, Gafsa & \\
\hline $\begin{array}{l}\text { Daucus hybrid } \\
\text { (D. carota, D. capillifolius) }\end{array}$ & Ames 30253 & 2010 & Tunisia, Nabuel & \\
\hline D. involucratus & PI 652350 & 2011 & Turkey, Izmir & \\
\hline D. littoralis & PI 295857 & 2010 and 2011 & Israel, Beit Alpha & \\
\hline
\end{tabular}

${ }^{\dagger}$ Complete locality data can be obtained at www.ars-grin.gov/.

fWe agree with the identifications for the accessions for which this column is left blank. 
Table 2. The 36 morphological characters measured in this study, modeling type, and F-test $p$ values of characters retained in a stepwise discriminant analysis for 1: all accessions identified as in Table 1; 2: a subset of the accessions containing and reidentified as $D$. capillifolius, $D$. carota subsp. gummifer, and $D$. carota subsp. carota; and 3: all accessions of $D$. capillifolius and $D$. carota (all subspecies) designated as one group and all other Daucus designated as another group.

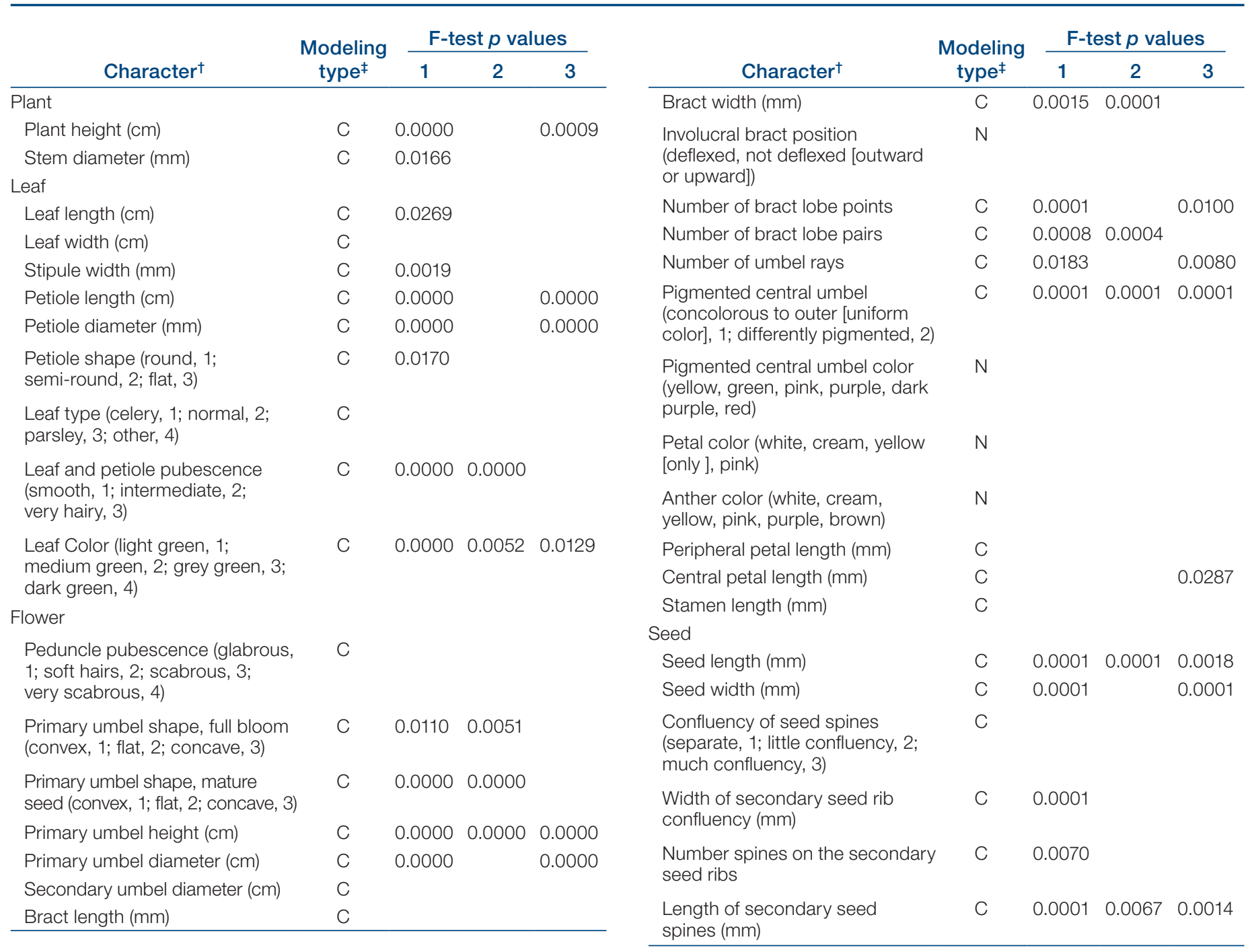

\section{RESULTS}

\section{Phenetic Analyses}

${ }^{\dagger}$ Additional details on these descriptors can be found in IPGRI (1998).

${ }^{\ddagger} \mathrm{N}$, nominal; $\mathrm{C}$, continuous.

Hierarchical cluster analyses using all data with the five types of distance methods (analyses not shown) failed to group the subspecies as initially identified. Stepwise discriminant analyses, however, aided the reidentifications of some specimens. Some were changed from one subspecies to another within $D$. carota, and some $D$. carota were changed to D. guttatus (Table 1). Reiterative analyses produced stable results only after $D$. carota was divided into $D$. carota subsp. carota sensu lato (in a broad or taxonomically expanded sense), and D. carota subsp. gummifer (also sensu lato). These two taxa correspond to Onno's (1937) D. gingidium and D. carota and to Small's (1978) and Reduron's (2007) two "species aggregates," or "subgroups," respectively, within the single species $D$. carota. Stepwise discriminant analysis was conducted for three subsets of the accessions, each using a different number of characters: Analysis 1 included all taxa examined for 23 continuously

variable morphological characters (F-test $p \leq 0.05$ ); Analysis 2 included just $D$. carota, all subspecies, and $D$. capillifolius in the $D$. carota clade analyzed for only 10 characters; Analysis 3 included all members of the D. carota clade as one group and all other accessions as a second group and analyzed 13 characters (Table 2).

Analysis 1 placed most specimens in three groups, as is evident from a canonical variates plot that shows the points and multivariate means in two dimensions that best separate the groups (Fig. 1). The first group corresponds to D. carota subsp. gummifer, the second group corresponds to D. carota subsp. carota sensu lato, and the third group includes D. broteri and D. guttatus. Daucus capillifolius and putative $D$. capillifolius $\times$ carota hybrids formed a group that partly overlaps with second group above, and the remaining taxa were scattered around the edge of the diagram. 


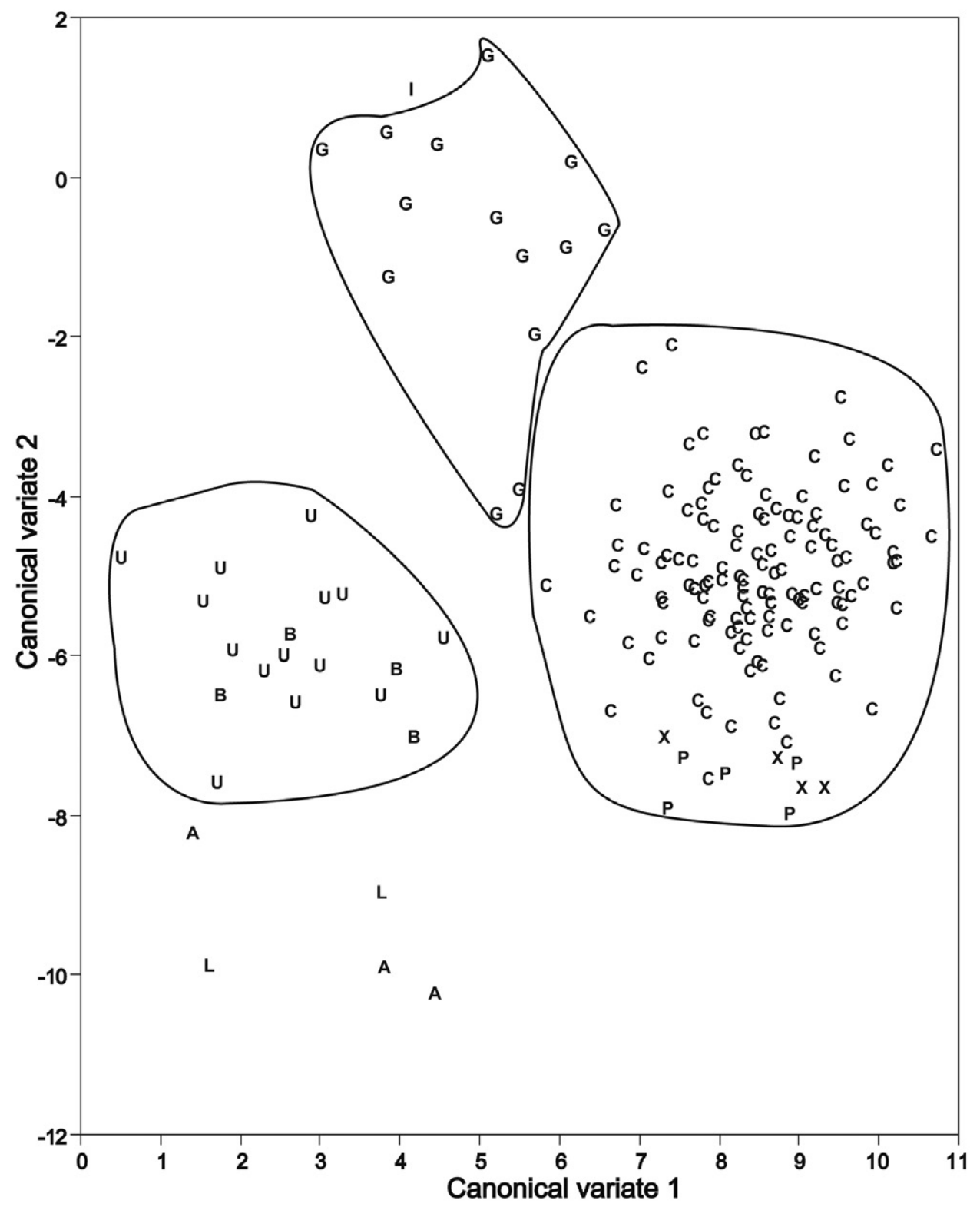

Figure 1. Plots of the first two canonical variates from discriminant analysis of all taxa based on the proposed new identifications of taxa (Table 1). A, Daucus aureus; B, D. broteri; C, D. carota subsp. carota; G, D. carota subsp. gummifer; I, D. involucratus; L, D. littoralis; P, D. capillifolius; U, D. guttatus; X, hybrids between D. capillifolius and D. carota, no subspecies.

Analysis 2 produced better separation of the $2 n=18$ species than did the analysis of all taxa (Fig. 2 and 3). The 15 replicate accessions examined in both 2010 and 2011 were all consistently assigned to their respective taxonomic groups. Analysis 3 clearly separated these two groups (Fig. 4; canonical variates analyses not shown). See Table 2 for $F$ values of all three analyses.

\section{Character-State Distributions}

An examination of the 10 strongest variable characters (all $p \leq 0.05$ ) separating $D$. carota subsp. carota, D. carota subsp. gummifer, and D. capillifolius accessions (Fig. 3), and these $2 n=18$ accessions compared with all other species (Fig. 4 ), shows considerable overlap within the character-state distributions supporting these groups. The best characters separated the $2 n=18$ species from the others on size characters (plant height, petiole length, primary umbel height and diameter) and number of plant parts (number of umbel rays), but with considerable overlap. An analysis within the $2 n=18$ accessions shows a similar pattern of character-state overlap, with the most obvious characters being the color of the central umbel (concolorous yellow in D. capillifolius; concolorous white, to pink to purple in all subspecies of $D$. carota), bract width (always $<15 \mathrm{~mm}$ in D. capillifolius, but sometimes also $<15 \mathrm{~mm}$ in D. carota), and seed length and secondary seed spine length (longest in $D$. capillifolius). Of these characters, only seed length separates the two species with little overlap.

The four nominal morphological characters (of 36 total; Table 2) are inappropriate for discriminant analyses, 


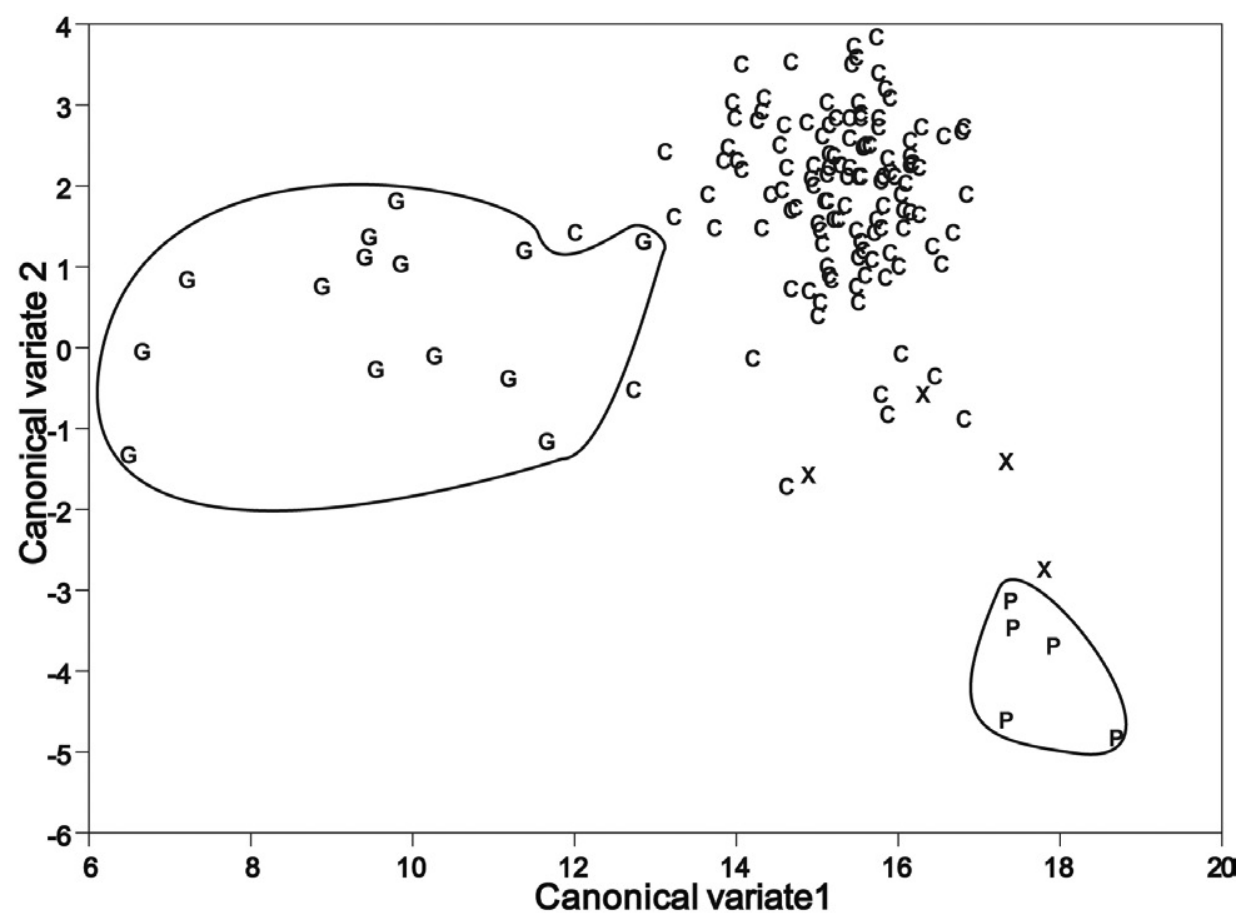

Figure 2. Plots of first two canonical variates from discriminant analysis of just the $2 n=18$ taxa based on the proposed new identifications of taxa (Table 1). C, Daucus carota subsp. carota; G, D. carota subsp. gummifer; P, D. capillifolius; X, hybrids between D. capillifolius and D. carota subsp. carota.

but three of them show clear separations of some taxa. Daucus capillifolius is the only taxon with yellow-pigmented central umbels and petal colors. Daucus capillifolius and D. carota subsp. gummifer have more involucral bracts pointed upward or outward than do the other taxa.

\section{DISCUSSION}

Our analysis, which is focused on representative accessions of $D$. carota subspecies and the related species D. capillifolius, with a few unrelated Daucus species for comparison, shows great overlap of character-state distributions among taxa. It highlights the great morphological similarity among these taxa for most characters and suggests that for wild $D$. carota only two subgroups can be separated morphologically. Morphological definition of even a limited number of subspecific taxa within $D$. carota, therefore, relies entirely on polythetic support, that is, grouping taxa that have the greatest number of shared features, no single feature of which is essential to group membership or is sufficient to make an organism a member of a group (Sokal and Sneath, 1963). Such concepts have been used in many complex groups exhibiting poorly defined isolating mechanisms but great within-group morphological variation, such as wild potatoes (Van den Berg et al., 1998) and indeed in many other difficult taxonomic groups.

The lack of agreement on circumscription of subspecies within $D$. carota, combined with the lack of a comprehensive taxonomic treatment of the subspecies throughout their ranges, has precluded stable and reliable identifications at the NCRPIS and other carrot gene banks. The best we can do at present with representative samples at the NCRPIS is to define two taxa, D. carota subsp. carota sensu lato and subsp. gummifer, corresponding to the two species (D. carota and D. gingidium) recognized by Onno (1937) or to the two "species aggregates," or "subgroups," recognized by Small (1978) and Reduron (2007) although without recognizing subspecies within these groups.

This study was designed to discover clear and practical methods to identify germplasm collections of $D$. carota and related species, but we await additional studies for a monographic level treatment of final taxonomic names. We attribute this to four factors requiring additional information. First, one of the characters used in the literature for D. carota subsp. gummifer, leaves that are stiff and shiny, was impossible to assess in an efficient manner because of so much pertinent phenotypic variation within and among accessions. Second, we found much variation in the subspecies or undescribed distinctive forms of $D$. carota. For example, in Tunisia, we identified two forms of $D$. carota, a typical form found in the United States and worldwide, identified as subsp. carota, and also another, which was encountered only in northwestern Tunisia and had inflated, leathery stipule bases and relatively large spherical umbels with tightly appressed and sclarified bracts in fruit. Further to the west, in Morocco, this was also a common morphotype (along with subsp. gummifer along the coast). However, these stipule-base and umbel characters vary greatly across Tunisia and Morocco, and it is difficult to assign collections to this morphotype. Third, the accessions that we evaluated in our study are not as comprehensive as we would have preferred, 


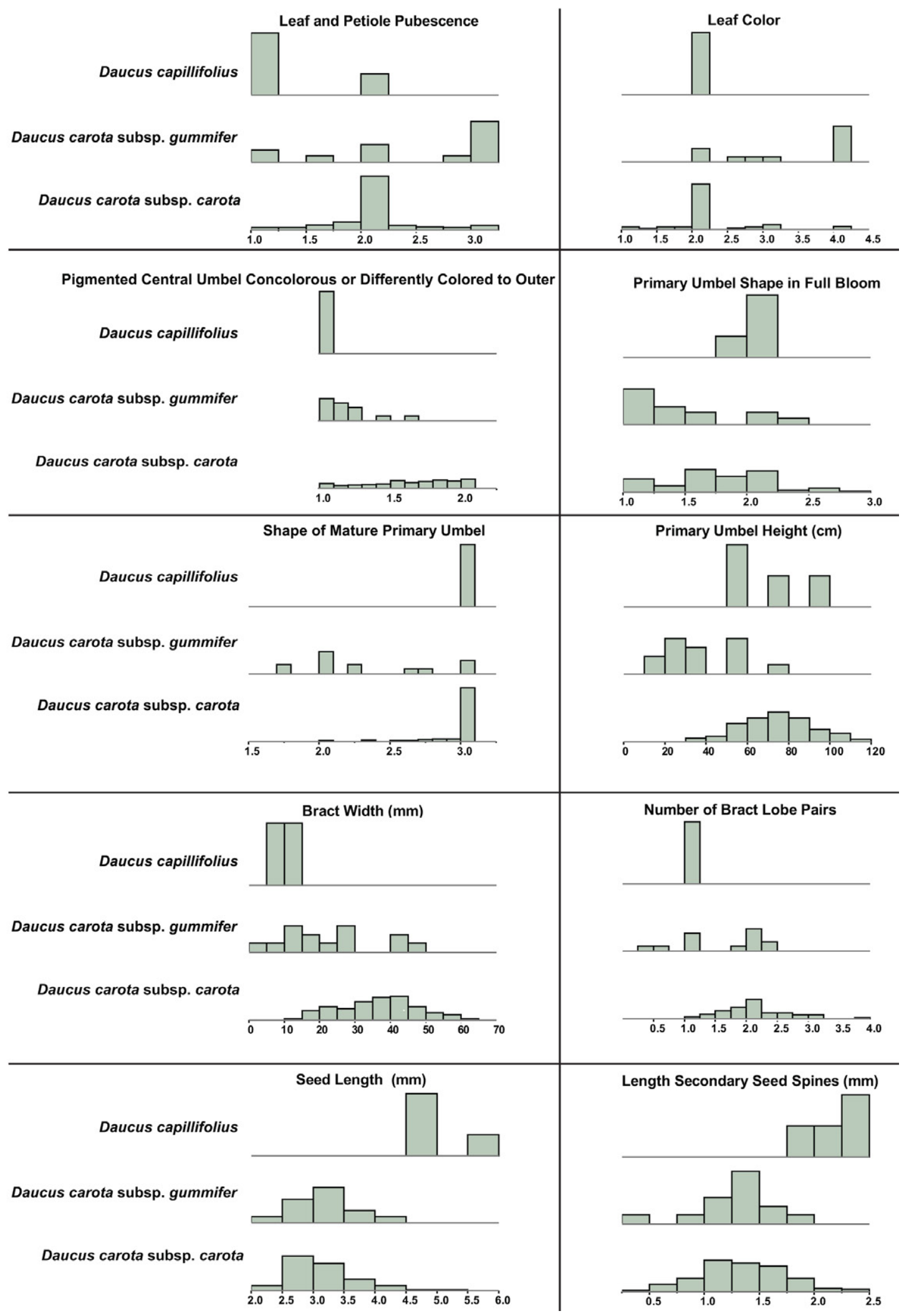

Figure. 3. Histograms of character-state distributions of the 10 strongest characters (Table 2) separating the $2 n=18$ taxa: $D$. capillifolius $(n=5)$, D. carota subsp. gummifer $(n=14), D$. carota subsp. carota and subsp. sativus $(n=126)$; excludes the three interspecific hybrids of $D$. carota $\times D$. capillifolius $(n=3)$ and other species $(n=22)$.

and we lack much of the subspecific variation described for the Iberian Peninsula by Pujadas Salvà (2003). Fourth, single nucleotide polymorphism examination of 81 accessions of cultivated and wild $D$. carota and closely related species (Iorizzo et al., 2013) was able to distinguish D. carota subspecies and even geographic subsets in subsp. carota better 


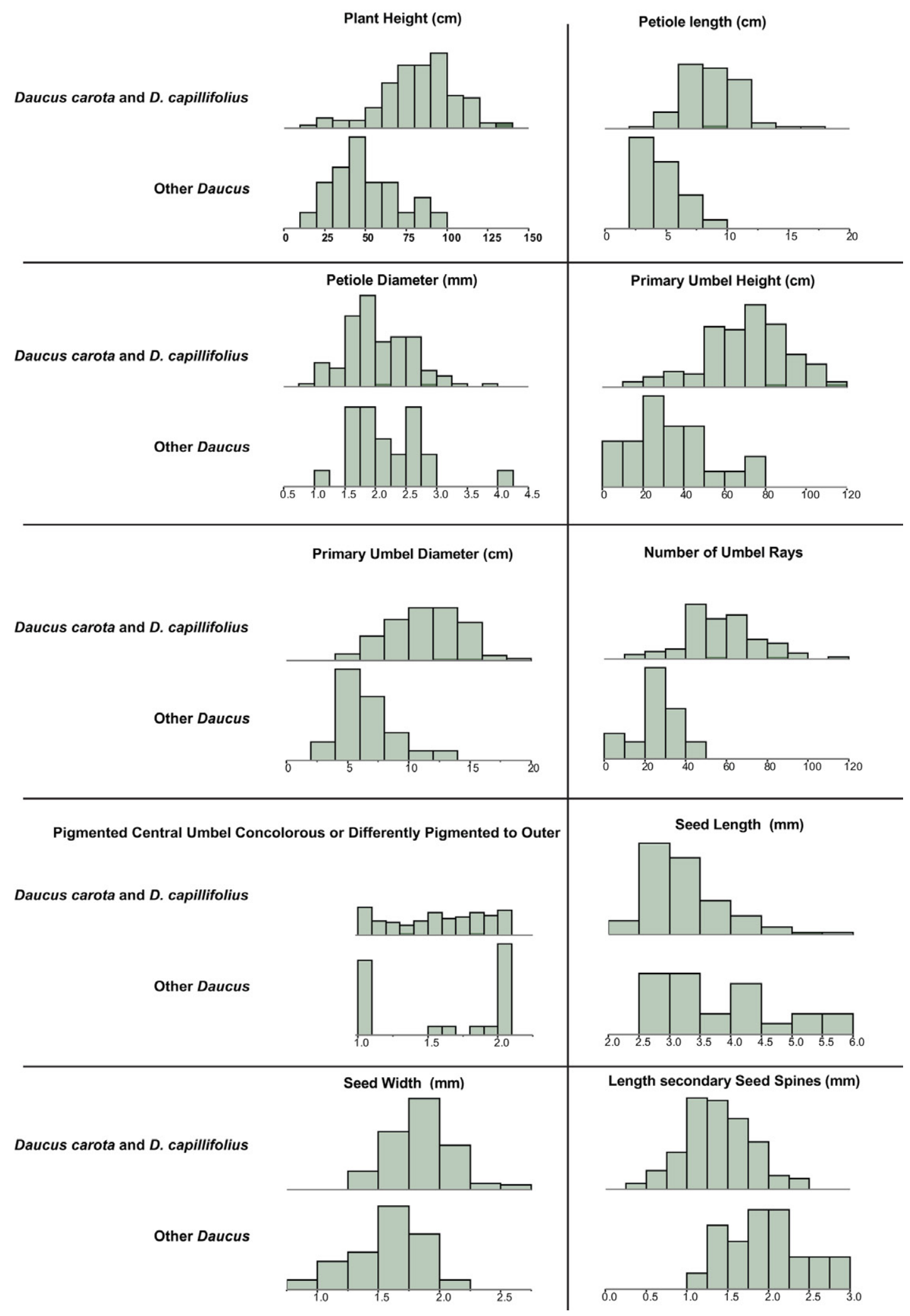

Figure. 4. Histograms of character-state distributions of the 10 strongest (of 13 total) characters (Table 2) separating Daucus capillifolius and D. $\operatorname{carota}(n=148)$ from all other Daucus $(n=22)$.

than our present study. Interestingly, Iorizzo et al. grouped $D$. capillifolius with $D$. carota subsp. carota, a result concordant with the DNA sequence data of multiple nuclear orthologs (Spooner et al., 2013) and with an amplified study using more accessions and nuclear orthologs (Arbizu et al., 2013), suggesting the need for a reclassification of $D$. capillifolius to $D$. carota, as suggested by the crossing studies of McCollum (1975). The phylogenetic studies of Spooner et al. (2013) and 
Arbizu et al. (2013), which used nuclear ortholog sequencing, also failed to distinguish even the two subgroupings of $D$. carota that we distinguish here. However, the same accessions were not always used in those molecular studies and the present morphological study. Our definitive conclusions await additional field experience and access to additional materials in different geographic regions for further morphological and molecular studies.

We analyzed accessions from many areas worldwide, with a concentration in the Mediterranean region, where $D$. carota is most diverse. Our proposed new identifications (Table 1) are of two main types: (i) those resulting from reduced numbers of taxa we support here, that is, $D$. carota subsp. carota sensu lato to include the names subsp. carota, subsp. major, subsp. maritimus, and subsp. maximus; and $D$. carota subsp. gummifer to include the names D. carota subsp. commutatus, subsp. drepanensis, subsp. gummifer, and subsp. hispanicus; (ii) unexpected identifications outside of these groups, including $D$. carota without subspecies to $D$. carota subsp. gummifer, or D. carota without subspecies designation and subsp. fontanesii to D. guttatus. Most proposed new identifications to subsp. gummifer are concentrated in the Mediterranean regions because these morphotypes are endemic there. Proposed new identifications of D. carota to D. guttatus are concentrated in Turkey, are unexpected, and require further morphological analyses of all available Daucus species (currently in progress).

At present, we will apply our morphological results as a basis for verification and possible reidentification of Daucus accessions in the GRIN database, noting that GRIN does retain former identifications to alert users of prior status. Our long-term plan is to use an integrated approach of morphological and molecular studies to clarify substructure in $D$. carota, as has been done in other groups such as cultivated potatoes (Spooner et al., 2007) and sorghum (Brown et al., 2011). However, we suspect that these additional studies will also conclude there are only two subspecies of $D$. carota.

\section{Acknowledgments}

This work was supported by USDA National Research Initiative grant no. 2008-35300-18669 to David Spooner and by USDA-ARS National Plant Germplasm System Horticultural Evaluation Grants to David Spooner and Philipp Simon. We gratefully acknowledge the collecting assistance by personnel of the Moroccan and Tunisian National Gene Banks and field assistance by Ms. Lucinda Clark and other NCRPIS staff. We gratefully acknowledge the review comments of John Wiersema and another unnamed reviewer. The use of trade, firm, or corporation names in this website is for the information and convenience of the reader. Such use does not constitute an official endorsement or approval by the United States Department of Agriculture or the Agricultural Research Service of any product or service to the exclusion of others that may be suitable.

\section{References}

Arbizu, C., H. Ruess, D. Senalik, P. Simon, and D. Spooner. 2013. Multiple nuclear ortholog next generation sequencing phylogeny of Daucus. Paper presented at: Botany 2013: Celebrating diversity. Botanical Society of America annual meeting, New Orleans, LA. 27-31 July. Paper 895. www.2013.botanyconference.org/engine/search/index.php?func=detail\&aid $=895$ (accessed 16 Jan. 2014).

Brown, P.J., S. Myles, and S. Kresovich. 2011. Genetic support for phenotype-based racial classification in sorghum. Crop Sci. 51:224-230. doi:10.2135/cropsci2010.03.0179

Constance, L. 1971. History of the classification of Umbelliferae (Apiaceae). In: V.H. Heywood, editor, The biology and chemistry of the Umbelliferae. Academic Press, New York. p. 1-8.

Cullen, J. 1972. Daucus. In: P.H. Davis, editor, Flora of Turkey and the East Aegean Islands. Vol. 4, Flora of Turkey. Edinburgh Univ. Press, Edinburgh, UK. p. 531-536.

Downie, S.R., D.S. Katz-Downie, and M.F. Watson. 2000. A phylogeny of the flowering plant family Apiaceae based on chloroplast DNA rpl16 and rpoc1 intron sequences: Towards a suprageneric classification of subfamily Apioideae. Am. J. Bot. 87:273-292. doi:10.2307/2656915

Ellis, P.R., J.A. Hardman, T.C. Crowther, and P.L. Saw. 1993. Exploitation of the resistance to carrot fly in the wild carrot species Daucus capillifolius. Ann. Appl. Biol. 122:79-91. doi:10.1111/j.1744-7348.1993.tb04016.x

Grzebelus, D., R. Baranski, K. Spalik, C. Allender, and P.W. Simon. 2011. Daucus. In: C. Kole, editor, Wild crop relatives: Genomic and breeding resources. Vol. 5, Vegetables. Springer Verlag, Berlin. p. 129-216.

Heywood, V.H. 1968. Daucus. Feddes Repert. 79:1-68.

Iovene, M., E. Grzebelus, D. Carputo, J. Jiang, and P.W. Simon. 2008. Major cytogenetic landmarks and karyotype analysis in Daucus carota and other Apiaceae. Am. J. Bot. 95:793-804. doi:10.3732/ajb.0700007

Iorizzo, M., D. Senalik, S. Ellison, D. Grzebelus, P. Cavagnaro, C. Allender, J. Brunet, D.M. Spooner, A. Van Deynze, and P.W. Simon. 2013. Genetic structure and domestication of carrot (Daucus carota subsp. sativus L.) (Apiaceae). Am. J. Bot. 100:930-938. doi:10.3732/ajb.1300055

IPGRI. 1998. Descriptors for wild and cultivated Carrots (Daucus carota L.). International Plant Genetic Resources Institute, Rome, Italy. www.ecpgr.cgiar.org/fileadmin/bioversity/ publications/pdfs/17_Descriptors_for_wild_and_cultivated_carrots__Daucus_carota_L._.pdf?cache $=1357818242$ (accessed 16 Jan. 2014).

Jafri, S.M.H., and A. El-Gadi. 1985. Daucus. In: S.M.H. Jafri and A. El-Gadi, editors, Apiaceae. Vol. 117 of Flora of Libya. Al Faatheh Univ., Faculty of Science, Dep. Botany, Tripoli, Libya. p. 130-144.

Jury, J.L. 2002. Daucus. In: B. Valdés, M. Rejdali, A. Achhal El Kadmiri, J.L. Jury, and J.M. Montserrat, editors, Catalogue des plantes vasculaires du Nord de Maroc, incluant des clés d'identification. Vol. 1. Consejo Superior de Investigaciones Cientiìficas, Madrid. p. 467-469.

Krickl, M. 1961. Karotten: Zur Frage der Verkreuzung mit der wilden Karotte. Saatgut-Wirtschaft 13:135-136.

Lee, B.Y., and S.R. Downie. 2000. Phylogenetic analysis of cpDNA restriction sites and rpsl6 intron sequences reveals relationships among Apiaceae tribes Caucalideae, Scandiceae and related taxa. Plant Syst. Evol. 221:35-60. doi:10.1007/BF01086379 
Le Floc'h, É., L. Boulos, and E. Véla. 2010. Catalogue synonymique commenté de la flore de Tunisie. République Tunisienne, Ministère de l'Environnement de Développment Durable Banque Nationale de Gènes, Tunis.

Magnussen, L.S., and T.P. Hauser. 2007. Hybrids between cultivated and wild carrots in natural populations in Denmark. Heredity 99:185-192. doi:10.1038/sj.hdy.6800982

McCollum, G.D. 1975. Interspecific hybrid Daucus carota $\times D$. capillifolius. Bot. Gaz. 136:201-206. doi:10.1086/336803

McCollum, G.D. 1977. Hybrids of Daucus gingidium with cultivated carrots (D. carota subsp. sativus) and D. capillifolius. Bot. Gaz. 138:56-63. doi:10.1086/336899

Mouterde, P. 1986. Nouvelle Flore de Liban et de la Syrie. Part 2. Dar El-Machreq Editeurs, Beirut, Lebanon.

Nehou, J. 1961. Recherches sur la taxonomie de genre Daucus (Ombellifères) en Bretagne. Bull. Soc. Sci. Bretagne 36:81-107.

Nothnagel, T., P. Straka, and B. Linke. 2000. Male sterility in populations of Daucus and the development of alloplasmic male-sterile lines of carrot. Plant Breed. 119:145-152. doi:10.1046/j.1439-0523.2000.00470.x

Onno, M. 1937. Die wildformen von Daucus sect. Carota. Beih. Bot. Zentralbl. 56(B):83-136.

Pimenov, M.G., and M.V. Leonov. 1993. The genera of Umbelliferae: A nomenclator. Royal Botanic Gardens, Kew, UK.

Plunkett, G.M., D.E. Soltis, and P.S. Soltis. 1996. Evolutionary patterns in Apiaceae: Inferences based on matK sequence data. Syst. Bot. 21:477-495. doi:10.2307/2419610

Pujadas Salvà, A.J. 2003. Daucus. In: G. Nieto Feliner, S.L. Jury, and A. Herrero, editors, Flora Iberica: Plantas vasculares de la península Ibérica e islas Baleares. Vol. 10, Alaliaceae-Umbelliferae. Real Jardín Botánico, CSIC, Madrid. p. 97-125.

Quezel, P., and S. Santa. 1963. Daucus. In: P. Quezel and S. Santa, editors, Nouvelle Flore de L'Algérie et regions désertiques méridionales. Éditions du Centre National de la Recherche Scientifique, Montpellier, France. p. 659-663.

Reduron, J.P. 2007. Ombellifères de France. Vol. 2. Société botanique du Centre-Ouest, Nercillac, France. 564 p.

Rubatzky, V.E., C.F. Quiros, and P.W. Simon. 1999. Carrots and related vegetable Umbelliferae. CABI Publishing, New York.
Sáenz Laín, C. 1981. Research on Daucus L. (Umbelliferae). Anales Jard. Bot. de Madrid. 37:481-534.

SAS Institute. 2010. JMP, Version 9.0.3. SAS Inst., Cary, NC.

Simon, P.W. 1984. Carrot genetics. Plant Mol. Biol. Rep. 2:54-63. doi:10.1007/BF02885649

Small, E. 1978. A numerical taxonomic analysis of the Daucus carota complex. Can. J. Bot. 56:248-276. doi:10.1139/b78-033

Sokal, R.R., and R.R. Sneath. 1963. Principles of numerical taxonomy. W. H. Freeman, San Francisco.

Spalik, K., and S.R. Downie. 2007. Intercontinental disjunctions in Cryptotaenia (Apiaceae, Oenantheae): An appraisal using molecular data. J. Biogeogr. 34:2039-2054. doi:10.1111/ j.1365-2699.2007.01752.x

Spooner, D.M., J. Núñez, G. Trujillo, M. del Rosario Herrera, F. Guzmán, and M. Ghislain. 2007. Extensive simple sequence repeat genotyping of potato landraces supports a major reevaluation of their gene pool structure and classification. Proc. Natl. Acad. Sci. USA 104:19398-19403. doi:10.1073/ pnas.0709796104

Spooner, D.M., P. Rojas, M. Bonierbale, L.A. Mueller, M. Srivastav, D. Senalik, and P. Simon. 2013. Molecular phylogeny of Daucus. Syst. Bot. 38:850-857. doi:10.1600/036364413X670449

Steinborn, R., B. Linke, T. Nothnagel, and T. Börner. 1995. Inheritance of chloroplast and mitochondrial DNA in alloplasmic forms of the genus Daucus. Theor. Appl. Genet. 91:632-638. doi:10.1007/BF00223290

Umiel, N., R. Jacobson, and D. Globerson. 1975. Pollination of the cultivated carrot (Daucus carota L.) by the wild carrot (D. carota var. maximus), and its implications on commercial seed production. (In Hebrew with English summary.) Hassadeh 56:478-480.

Van den Berg, R.G., J.T. Miller, M.L. Ugarte, J.P. Kardolus, J. Villand, J. Nienhuis, and D.M. Spooner. 1998. Collapse of morphological species in the wild potato Solanum brevicaule complex (sect. Petota). Am. J. Bot. 85:92-109. doi:10.2307/2446559

Wijnheijmer, E.H.M., W.A. Brandenburg, and S.J. Ter Borg. 1989. Interactions between wild and cultivated carrots (Daucus carota L.). Euphytica 40:147-154.

Zohary, M. 1972. Flora Palaestina. Part 2. Israel Academy of Sciences and Humanities, Jerusalem. 